\title{
Report on the Third Conference on Artificial General Intelligence
}

\author{
Ben Goertzel and Marcus Hutter
}

- The Third Conference on Artificial General Intelligence was held March 5-8, 2010, at the University of Lugano in Lugano, Switzerland. The conference was chaired by Marcus Hutter and cochaired by Eric Baum and Emanuel Kitzelmann. The conference also provided a venue for the awarding of the 2010 Kurzweil Prizes.
$\mathrm{D}$ uring March 5-8, 2010, around 75 researchers from various disciplines converged at the University of Lugano for the Third Conference on Artificial General Intelligence (AGI-10). The conference was dedicated to the memory of the recently deceased AI pioneer Ray Solomonoff, whose wife Grace attended the conference and gave a very moving presentation on Ray's life and work.

The AGI conferences are the only major conference series devoted wholly and specifically to the creation of AI systems possessing general intelligence at the human level and ultimately beyond. Sponsored by AAAI, KurzweilAI.net, and the Università della Svizzera Italiana, AGI-10 was the first European AGI conference and continued the mission of the first two AGI conferences held in the USA: gathering an international group of leading academic and industry researchers involved in serious scientific and engineering work aimed directly toward the goal of creating AI systems with general intelligence at the human level and ultimately beyond. The participants spanned a host of fields including computer scientists, philosophers, psychologists, economists, neuroscientists, mathematicians, physicists, and more.

The tone of the gathering was optimistic, reflecting the attitude captured by a survey taken of the AGI-09 conference (published in $H+$ Magazine), ${ }^{1}$ which showed that a significant plurality of AGI researchers consider it likely human-level AGI will come within a few decades. The general feeling among presenters seemed to be that, though much work still remains, 


\section{ICWSM-11 to be Collocated with IJCAI-11 in Barcelona, Spain!}

T he Fifth International AAAI Conference on Weblogs and Social Media will be held in in Barcelona, Spain in July 2011. This interdisciplinary conference brings together researchers and industry leaders interested in creating and analyzing social media. Past conferences have included technical papers from areas such as computer science, linguistics, psychology, statistics, sociology, multimedia and semantic web technologies. A full Call for Papers will be available this fall at www.icwsm.org, and papers will be due in mid-January 2011. As in previous conferences, collections of social-media data will be provided by ICWSM-11 organizers to potential participants to encourage experimentation on common problems and datasets. For more information, please write to icwsm11@ aaai.org. design principle that is thought by many AGI researchers to be one of the keys to achieving practical AGI. The paper also describes a real-world test problem, the Toy Box problem (involving flexibly utilizing a variety of toys in a preschool-type toy box), which serves as a natural ground for describing and testing Comirit and other AGI architectures.

The dichotomy between these two prize-winning papers illustrates one of the key threads of discussion that ran through the conference. On the one hand, a number of researchers presented abstract mathematical approaches to AGI (the conference by the Sun et al. paper, Marcus Hutter's tutorial, and others). On the other hand, the more pragmatic system-building approach to AGI was also well represented - by Johnston, SOAR pioneer John Laird, Ben Goertzel, and others. While these two approaches are ultimately aimed at the same goal, the relation between them is not always clear. One idea that surfaced in several presentations and discussions was that the architectural principles underlying pragmatic AGI systems seem to reflect adaptation to properties of real-world environments, which are not yet captured in the formal models used by those pursuing rigorous AGI.

The final day of the conference included a workshop led by Selmer Bringsjord and Marcus Hutter on AGI as a rigorous science; and a workshop led by Ben Goertzel on the future of AGI and roadmaps to AGI, which included a summary of the AGI Roadmap Workshop held at the University of Tennessee in October 2009, and also a talk by philosopher Anders Sandberg from Oxford University's Future of Humanity Institute on AGI and possible paths to technological singularity.

While the community of AGI researchers is nowhere near a consensus on the best approach to the original, grand goal of the AI field, it's clear that the pursuit of the goal is alive and well, and yielding interesting discoveries and discussions.

The AGI-11 conference is planned for early August 2011 in the San Francisco Bay area.

\section{Note}

1. See bit.ly/hplus-ai-survey.

Best AGI Paper went to "Frontier Search" by Lugano researchers Yi Sun, Tobias Glasmachers, Tom Schaul, and Juergen Schmidhuber, which presents a novel formal framework unifying earlier, Solomonoff-induction-inspired approaches to rigorous AGI such as Levin search and the speed prior.

The Kurzweil Prize for Best AGI Idea was awarded to "The Toy Box Problem (and a Preliminary Solution)" by Australian Ph.D. student Benjamin Johnston. Johnston's Comirit architecture combines inference and simulation in an intimate way, thus embodying the integrative
Ben Goertzel, Ph.D., is the chief executive officer of Novamente LLC. Goertzel is the author of more than 70 research papers, journalistic articles, and scholarly books dealing with topics in the cognitive sciences and futurism. He is the principal architect of the Novamente Cognition Engine.

Marcus Hutter, Ph.D., is an associate professor at the RSISE at the Australian National University in Canberra, Australia. He is also a NICTA adjunct. His research is centered on the information-theoretic foundations of inductive reasoning and reinforcement learning. 